\title{
Validade de conteúdo nos processos de construção e adaptação de instrumentos de medidas
}

\author{
Content validity in the development \\ and adaptation processes of measurement instruments
}

Neusa Maria Costa Alexandre ${ }^{1}$

Marina Zambon Orpinelli Coluci ${ }^{1}$

${ }^{1}$ Departamento de

Enfermagem, Faculdade de Ciências Médicas,

Universidade Estadual de Campinas. Cidade Universitária Zeferino Vaz, Barão Geraldo. 13083-970 Campinas SP.

nesalex@fcm.unicamp.br

\begin{abstract}
This study sought to conduct a review of content validity, which is an important phase of processes of construction and adaptation of measurement instruments. Research of comprehensive literature was conducted by means of a review of national and international databases. Initially, a description of the conceptual basis and the measurement methods used in content validity was made, with emphasis on its application in the healthcare area. It was seen that controversy exists in the literature on the terminology and concept of content validity. The recommended procedures used to check content validity during the construction and adaptation processes of instruments were described, especially the judges' assessment, which can involve qualitative and quantitative procedures. The number, selection and qualification of these judges were also described. The different methods used to quantify the level of agreement among the experts were verified, mainly the Content Validity Index (CVI). This study described aspects of the content validity process, one of the procedures to be considered by healthcare researchers and professionals who are interested in using reliable and appropriate measurements and instruments scales for given population groups.

Key words Validity of tests, Validation studies, Questionnaires, Methods, Reproducibility of results
\end{abstract}

Resumo Este estudo teve por objetivo realizar uma revisão sobre validade de conteúdo, fase importante nos processos de construção e adaptação de instrumentos de medida. Foi realizada pesquisa bibliográfica definida como integrativa, por meio de pesquisa a bases de dados nacionais e internacionais. Iniciou-se a descrição das bases conceituais e os métodos de medidas usados em validade de conteúdo, com ênfase na área da saúde. Verificou-se que existem controvérsias na literatura sobre a terminologia e o conceito da validade de conteúdo. Foram descritos os procedimentos recomendados para realizar a validade de conteúdo durante os processos de construção e de adaptação de instrumentos, particularmente a avaliação por juízes, o que pode envolver procedimentos qualitativos e quantitativos. Descreveu-se número, seleção e qualificação desses juízes. Os diferentes métodos para quantificar o grau de concordância entre os especialistas foram analisados, principalmente o Índice de Validade de Conteúdo (IVC). Este estudo descreveu aspectos do processo de realização da validade de conteúdo, um dos procedimentos a serem considerados por pesquisadores e profissionais da área de saúde preocupados em utilizarem medidas e instrumentos confiáveis e apropriados para determinada população. Palavras-chave Validade dos testes, Estudos de validação, Questionários, Métodos, Reprodutibilidade dos testes 


\section{Introdução}

Ao planejar o método de coleta de dados, devese pensar em procedimentos que garantam indicadores confiáveis. A decisão vai depender do desenho da pesquisa e da seleção de instrumentos de medidas adequados e precisos. Um número crescente de questionários e escalas está atualmente disponível na área de saúde.

Dessa forma, cada vez mais a literatura tem alertado os pesquisadores sobre uma correta avaliação das qualidades dos instrumentos de coleta de dados ${ }^{1-3}$. Os mais importantes atributos desses instrumentos são: validade (validity), confiabilidade (reliability), praticabilidade (usability), sensibilidade (sensitivity) e responsividade (responsiveness) ${ }^{4-6}$. Na realidade, as características de validade e de confiabilidade são particularmente importantes ao se escolher, desenvolver ou realizar a adaptação cultural de instrumentos que serão usados tanto em pesquisas como na prática clínica ${ }^{5,7}$.

$\mathrm{Na}$ área de ciências sociais, o conceito de validade deve ser aplicado em todas as medidas como inventários de personalidades, testes educacionais, surveys, questionários de atitudes, entre outros ${ }^{10}$. Deve também fazer parte da avaliação de instrumentos usados na clínica como entrevistas, exame físico, exames de laboratório, radiológicos e histológicos ${ }^{5}$.

A validade verifica se o instrumento mede exatamente o que se propõe a medir ${ }^{11}$. Isto é, avalia a capacidade de um instrumento medir com precisão o fenômeno a ser estudado ${ }^{12}$. Pode-se considerar um instrumento válido quando ele consegue avaliar realmente seu objetivo, como, por exemplo, ansiedade e não outro constructo como o estresse ${ }^{13}$. Pode ser avaliada por meio dos seguintes métodos: validade de conteúdo, validade relacionada a um critério e validade do constructo.

A confiablidade é a capacidade em reproduzir um resultado de forma consistente no tempo e no espaço, ou com observadores diferentes ${ }^{12}$. Os seguintes procedimentos são utilizados para sua avaliação: estabilidade (teste-reteste), homogeneidade e equivalência (inter-observadores) ${ }^{14}$.

Sensibilidade é a habilidade de a medida identificar diferenças entre pacientes ou grupo de pacientes com afecções leves e severas ${ }^{6}$. A responsividade avalia a capacidade do instrumento em detectar melhora ou piora nas condições de pacientes, isto é, consegue verificar mudanças ${ }^{6}$. A praticabilidade refere-se aos aspectos práticos da utili- zação de instrumentos. Podem-se levar em conta a facilidade e o tempo de administração, a interpretação dos resultados e os custos envolvidos 4 .

Estudos e discussões sobre essa temática são escassos particularmente em países da América Latina e Caribe, apesar de já se notar a utilização de avaliações psicométricas no desenvolvimento de instrumentos na área de saúde em países em desenvolvimento ${ }^{15,16}$.

Considera-se relevante a divulgação e a discussão dos diferentes métodos de se avaliar as propriedades psicométricas de instrumentos de coleta de dados utilizados na área de saúde, tanto na assistência como na pesquisa. Dessa forma, o objetivo do presente estudo foi realizar uma revisão sobre a validade de conteúdo.

\section{Método}

Trata-se de uma pesquisa bibliográfica que pode ser definida como integrativa ${ }^{17}$. Para o levantamento bibliográfico, optou-se pelas bases de dados das Ciências da Saúde como a Biblioteca Virtual em Saúde - Enfermagem (BDENF) e o Portal de Revistas de Enfermagem; o SCIELO; a LILACS (Literatura Latino - Americana e do Caribe em Ciências da Saúde); a MEDLINE (National Library of Medicine-USA); o International Nursing Index (INI) e o Cumulative Index to Nursing and Allied Health Literature (CINAHL).

Para seleção das pesquisas, foram utilizados alguns critérios. O tipo de publicação considerado foram os periódicos e os livros de metodologia de pesquisa e estatística. A procedência e o idioma deveriam ser de artigos nacionais e internacionais, publicados em português e inglês.Os descritores em português pesquisados na BIREME (DeCS - Terminologia em Saúde) foram: estudos de validação; tradução; validade dos testes e reprodutibilidade dos testes. Em inglês, a pesquisa foi feita na National Library of Medicine's Medical Subject Headings ( $\mathrm{MeSH}=$ Medical Subject Heading Terms), utilizando-se: validation studies; translations e psychometrics.

\section{Discussão}

Essa revisão foi organizada de modo a apresentar as bases conceituais e os métodos de medidas usados em validade de conteúdo, com ênfase em sua aplicação na área da saúde. 
Encontram-se controvérsias na literatura sobre a terminologia e o conceito da validade de conteúdo ${ }^{10,19}$. Para alguns autores, consiste em julgar em que proporção os itens selecionados para medir uma construção teórica representam bem todas as facetas importantes do conceito a ser medido ${ }^{12}$. Refere-se em verificar a extensão em que os itens de uma medida determinam o mesmo conteúdo ${ }^{20}$. Outros autores tentam explicar dessa forma: as perguntas do instrumento são representativas dentro do universo de todas as questões que poderiam ser elaboradas sobre esse tópico? ${ }^{21}$.

A validade de conteúdo é também definida de uma forma mais abrangente. Isto é, avalia o grau em que cada elemento de um instrumento de medida é relevante e representativo de um específico constructo com um propósito particular de avaliação ${ }^{19}$. Segundo os mesmos autores, os elementos de um instrumento são todos os aspectos do processo de medida que podem afetar a coleta de dados. No caso de um questionário, consideram-se como elementos as instruções, o formato das respostas e os itens de forma individual.

A validade de conteúdo é fundamental no processo de desenvolvimento e adaptação de instrumento de medidas ${ }^{10}$. No entanto, apresenta limitações por ser um processo subjetivo. Dessa forma, sua utilização não elimina a necessidade de aplicação de outras medidas psicométricas adicionais ${ }^{20}$.

\section{Abordagens para realizar a validade de conteúdo}

\section{Construção e adaptação cultural de instrumentos de medida}

Nessa fase, abordam-se inicialmente os procedimentos recomendados para realizar a validade de conteúdo durante os processos de construção e de adaptação de instrumentos de medida.

Para alguns autores, a validade de conteúdo compreende somente a avaliação por um comitê de especialistas ${ }^{4,7}$. No entanto, pesquisadores têm descrito que a validade de conteúdo é um processo de julgamento sendo composto por duas partes distintas. A primeira envolveria o desenvolvimento do instrumento e, posteriormente, a avaliação desse por meio da análise por especia$\operatorname{listas}^{22,23}$. Assim, pode-se considerar que a validade de conteúdo de instrumentos seria também garantida pelo procedimento de elaboração dos mesmos $^{24}$. Para avaliar a validade de conteúdo recomenda-se também utilizar procedimentos quantitativos e qualitativos ${ }^{19}$.

Para construir a variedade de itens, o pesquisador deve inicialmente definir o constructo de interesse e suas dimensões por meio de pesquisa bibliográfica e consulta a estudiosos da área e a representantes da população de interesse ${ }^{14,25}$. Autores têm defendido que esse estágio de desenvolvimento de instrumentos deve englobar três fases: identificação dos domínios, a formação dos itens e a construção do instrumento ${ }^{23,26-28}$. A fase de elaboração de questionários e escalas tem sido objeto de publicações específicas indicando diferentes metodologias ${ }^{29-31}$.

Autores sugerem e descrevem etapas e métodos padronizados e sistemáticos que deveriam ser usados durante esse processo com o objetivo de melhorar a qualidade dos instrumentos de medidas $^{19}$. Essas etapas diferem dependendo do que o instrumento quer avaliar. Segundo Lynn ${ }^{23}$, para medidas cognitivas deve-se seguir os seguintes passos: identificação da totalidade dos domínios; construção dos itens e por fim, organização desses itens.Ainda pensando no processo de delineamento dos itens, não se pode deixar de mencionar a questão da adaptação cultural de instrumentos de medidas ${ }^{31}$. A adaptação de um instrumento para outra linguagem é um processo complexo. A realização de uma simples tradução não pode ser efetuada devido às diferenças culturais e de linguagem. Deve-se levar em consideração o idioma, o contexto cultural e o estilo de vida. Assim, para realizar uma adaptação de um instrumento de uma linguagem para outra, deve-se levar em conta aspectos técnicos, linguísticos e semânticos ${ }^{32}$. A literatura internacional tem procurado propor normas para assegurar adaptações de instrumentos de uma forma metodologicamente correta. A tendência atual é padronizar normas internacionais para assegurar a qualidade de instrumentos adaptados. Segundo esses protocolos, os seguintes passos são essenciais nesse processo: tradução inicial; síntese; tradução de volta para a língua original; revisão por um comitê e realização de um pré-teste ${ }^{33-37}$.

\section{Avaliação por um comitê de especialistas}

Alguns autores defendem que o segundo estágio do procedimento da validade de conteúdo consiste na avaliação do instrumento por especi- 
alistas. Procura-se abordar esse procedimento no processo de construção de questionários e escalas e durante a realização de uma adaptação cultural.

Durante o desenvolvimento de instrumento, um dos pontos discutidos nessa avaliação é o número e a qualificação desses juízes. A literatura apresenta controvérsias sobre esse ponto. Lynn $^{23}$ recomenda um mínimo de cinco e um máximo de dez pessoas participando desse processo. Outros autores sugerem de seis a vinte sujeitos, sendo composto por um mínimo de três indivíduos em cada grupo de profissionais selecionados para participar ${ }^{19}$. Nessa decisão, devese levar em conta as características do instrumento, a formação, a qualificação e a disponibilidade dos profissionais necessários ${ }^{23,27}$.

Em relação à seleção, deve-se levar em consideração a experiência e a qualificação dos membros desse comitê. Recomenda-se, descrever os critérios utilizados nessa seleção ${ }^{23}$. Entre esses critérios, a literatura destaca: ter experiência clínica; publicar e pesquisar sobre o tema; ser perito na estrutura conceitual envolvida e ter conhecimento metodológico sobre a construção de questionários e escalas ${ }^{27,38}$. Indica-se também a inclusão de pessoas leigas potencialmente relacionadas com a população do estudo ${ }^{20,39}$. A inclusão de pessoas leigas asseguraria a correção de frases e termos que não estão muito claros.

Quando for um processo de adaptação cultural, sugere-se a formação de um comitê multidisciplinar ${ }^{35}$. Neste caso, recomenda-se que deve ser composto por pessoas bilíngües e por especialistas na área de conhecimento do instrumento, que precisam ser informados sobre as medidas e conceitos envolvidos ${ }^{33}$. Pode também ser composto por profissionais da área de saúde, professor de línguas, especialista da metodologia e pelos tradutores envolvidos no processo ${ }^{37}$. Alguns autores recomendam a inclusão de pessoas leigas ${ }^{32,34}$. Quando se tratar de adaptação entre culturas por população imigrante, sugere-se que indivíduos representativos dessa população estejam envolvidos.

A avaliação por juízes pode envolver procedimentos qualitativos e quantitativos ${ }^{14,39,40}$. O processo deve ser iniciado com o convite aos membros do comitê de juízes. Esses especialistas devem receber uma carta explicativa e um questionário desenvolvido especificamente para essa avaliação ${ }^{27}$. Alguns autores sugerem a inclusão de um questionário para uma breve caracterização desses especialistas ${ }^{20}$.

A carta deve explicar porque o sujeito foi escolhido como juiz e a relevância dos conceitos envolvidos e do instrumento como um todo ${ }^{23,27}$. Recomenda-se também incluir o objetivo do estudo, a descrição do instrumento e a sua pontuação e interpretação, e uma explicação sobre a forma de resposta ${ }^{20,38}$. Pode conter também definições conceituais que deram origem ao instrumento, as dimensões envolvidas e o modelo de medida usado. Isto é, fornecer as bases conceituais e teóricas do instrumento ${ }^{38}$. Se for importante, pode também incluir informações sobre o contexto e a população envolvida. Essa carta deve considerar o nível educacional dos membros do comitê quando incluir sujeitos leigos ${ }^{20}$.

Berk $^{28}$ relata que essa avaliação deve ser feita em dois momentos distintos, com orientações específicas para cada fase. Primeiro sugere realizar uma avaliação na fase de especificação dos domínios e depois na fase de desenvolvimento dos itens. De uma forma geral, recomenda-se que os especialistas recebam instruções específicas sobre como avaliar cada item, o instrumento como um todo e como preencher o questionário que orienta a avaliação ${ }^{14}$.

Os juízes devem inicialmente avaliar o instrumento como um todo, determinando sua abrangência. Isto é, se cada domínio ou conceito foi adequadamente coberto pelo conjunto de itens e se todas as dimensões foram incluídas ${ }^{39}$. Nesta fase, podem sugerir a inclusão ou a eliminação de itens ${ }^{20}$.

Precisam também analisar os itens individualmente verificando sua clareza e pertinência. Em relação à clareza, deve-se avaliar a redação dos itens, se eles foram redigidos de forma que o conceito esteja compreensível e se expressa adequadamente o que se espera medir ${ }^{27}$. Pertinência ou representatividade significa notar se os itens realmente refletem os conceitos envolvidos, se são relevantes e, se são adequados para atingir os objetivos propostos ${ }^{27,41}$. Deve-se deixar um espaço para que os especialistas possam redigir sugestões para melhorar o item ou fazer comentários ${ }^{20,39}$.

Essa avaliação pode ser inicialmente feita de forma individual e independente pelos juízes, seguida por uma discussão em grupo que corresponde ao procedimento qualitativo ${ }^{28,39,40}$. Alguns autores também defendem um processo interativo entre pesquisadores e os membros do comitê, sugerindo o emprego de entrevistas e discussões para clarificar pontos controversos ${ }^{27}$. A abordagem de se usar pelo menos dois métodos, geralmente quantitativo e qualitativo, é denominada triangulação metodológica ${ }^{42}$.

No caso de adaptação cultural, esse comitê deve assegurar que a versão final seja totalmente 
compreensível e avaliar a sua equivalência cultural. Há a necessidade de existir equivalências semântica, idiomática, conceitual e experimental ${ }^{34}$.

Equivalência semântica é relativa ao significado das palavras (vocabulário, gramática); equivalência idiomática refere-se às expressões idiomáticas e coloquiais (por exemplo, sentir-se em casa); equivalência experimental aborda situações coerentes com o contexto cultural (por exemplo: usar o carro / usar o transporte público; dificuldade para usar um garfo); e, finalmente, a equivalência conceitual se refere ao conceito explorado (por exemplo: explorar as maneiras que as diferentes populações entendem saúde ${ }^{33}$. Dentro deste contexto, o comitê pode modificar as orientações iniciais e o formato do instrumento, alterar ou rejeitar itens inadequados e até desenvolver novos itens. Para verificar se o instrumento está realmente em uma linguagem compreensível para uma grande parte de pessoas, recomenda-se que ele precisa ser entendido por indivíduos de 10 a $14 \operatorname{anos}^{33}$.

\section{Medidas quantitativas para avaliar validade de conteúdo}

Publicações têm apresentado métodos diferentes para quantificar o grau de concordância entre os especialistas durante o processo de avaliação da validade de conteúdo de um instrumento. Dentre esses, destaca-se:

Porcentagem de concordância

Método empregado para calcular a porcentagem de concordância entre os juízes. É a medida mais simples de concordância interobservadores ${ }^{43}$. A fórmula utilizada está descrita a seguir ${ }^{39,44}$.

$$
\% \text { concordância }=\frac{\begin{array}{c}
\text { número de } \\
\text { participantes que } \\
\text { concordaram }
\end{array}}{\begin{array}{c}
\text { número total de } \\
\text { participantes }
\end{array}} \times 100
$$

As vantagens desse procedimento é proporcionar informações úteis que são facilmente calculadas. No entanto, apresenta limitações que restringem sua utilização ${ }^{44}$. Autores têm usado esse método na fase inicial para auxiliar na determinação dos itens ${ }^{27,39}$. Ao usar esse método, deve-se considerar como uma taxa aceitável de concordância de $90 \%$ entre os membros do comitêt $\hat{2}^{22,44}$.

\section{Índice de validade de conteúdo (IVC)}

Compreende um método muito utilizado na área de saúde $e^{40,41,45}$. Mede a proporção ou porcentagem de juízes que estão em concordância sobre determinados aspectos do instrumento e de seus itens. Permite inicialmente analisar cada item individualmente e depois o instrumento como um todo. Este método emprega uma escala tipo Likert com pontuação de um a quatro. Para avaliar a relevância/representatividade, as respostas podem incluir: 1 = não relevante ou não representativo, 2 = item necessita de grande revisão para ser representativo, $3=$ item necessita de pequena revisão para ser representativo, 4 $=$ item relevante ou representativo ${ }^{20,23}$. Outros autores sugerem opções mais curtas. Por exemplo: 1 = não claro, 2 = pouco claro, 3 = bastante claro, $4=$ muito claro $^{18,25,40}$. Outra opção seria: $1=$ irrelevante a $4=$ extremamente relevante ${ }^{25}$.

O escore do índice é calculado por meio da soma de concordância dos itens que foram marcados por " 3 " ou " 4 " pelos especialistas ${ }^{27}$. Os itens que receberam pontuação " 1 ” ou " 2 " devem ser revisados ou eliminados. Dessa forma, o IVC tem sido também definido como "a proporção de itens que recebe uma pontuação de 3 ou 4 pelos juizes" ${ }^{18}$. A fórmula para avaliar cada item individualmente fica assim:

$$
\begin{gathered}
\text { número de respostas } \\
\text { " } 3 \text { " ou " } 4 \text { " }
\end{gathered}
$$

Para avaliar o instrumento como um todo, não existe um consenso na literatura. Polit e Beck ${ }^{22}$ recomendam que os pesquisadores devem descrever como realizaram o cálculo. Esses autores apresentam três formas que podem ser usadas. Uma é definida como a "média das proporções dos itens considerados relevantes pelos juizes". A outra é a "média dos valores dos itens calculados separadamente, isto é soma-se todos os IVC calculados separadamente e divide-se pelo número de itens considerados na avaliação". Finalmente, a última forma seria dividir o "número total de itens considerados como relevantes pelos juizes pelo número total de itens”. Deve-se também estipular a taxa de concordância aceitável entre os juízes. Autores defendem que no processo de avaliação dos itens individualmente, deve-se considerar o número de juizes. Com a participação de cinco ou menos sujeitos, todos devem concordar para ser representativo. No caso de seis ou 
mais, recomenda-se uma taxa não inferior a $0,78^{22,23}$. Para verificar a validade de novos instrumentos de uma forma geral, alguns autores sugerem uma concordância mínima de $0,80^{27,38}$. No entanto, neste caso os valores recomendados devem ser de 0,90 ou mais ${ }^{22}$.

\section{Coeficiente de kappa}

O coeficente de kappa ( $\mathrm{k}$ ) também tem sido recomendado para avaliar medidas de concordância entre avaliadores na área de saúde ${ }^{44,45}$. O coeficiente de kappa de concordância é a razão da proporção de vezes que os juizes concordam (corrigido por concordância devido ao acaso) com a proporção máxima de vezes que os juizes poderiam concordar (corrigida por concordância devido ao acaso $)^{43,46}$. É um índice útil quando vários avaliadores categorizam cada grupo de objetos ou sujeitos em categorias nominais. Des- sa forma, é aplicável quando os dados são categóricos e estão em uma escala nominal ${ }^{46}$. Os valores de kappa variam de -1 (ausência total de concordância) a 1 (concordância total $)^{43}$.

\section{Conclusão}

Este estudo descreve aspectos do processo de realização da validade de conteúdo, uma fase importante do desenvolvimento e adaptação de questionários e escalas. Ressalta-se que esse é somente o início de um processo que deve englobar também outras medidas para avaliar outros tipos de validade e a confiabilidade.

Compreender esses procedimentos é essencial para pesquisadores e profissionais da área de saúde preocupados em utilizar cada vez mais medidas e instrumentos confiáveis e apropriados para determinada população.

\section{Colaboradores}

NMC Alexandre e MZO Coluci participaram igualmente de todas as etapas de elaboração do artigo. 


\section{Referências}

1. Marx RG, Bombardier C, Hogg-Johnson S, Wright JG. Clinimetric and psychometric strategies for development of a health measurement scale. J Clin Epidemiol 1999; 52(2):105-111.

2. Selby-Harrington ML, Mehta SM, Jutsum V, Riportella-Muller R, Quade D. Reporting of instrument validity and reliability in selected clinical nursing journals. J Prof Nurs 1994; 10(1):47-56.

3. Salmond SS. Evaluating the reliability and validity of measurement instruments. Orthop Nurs 2008; 27(1):28-30.

4. Dempsey PA, Dempsey AD. Using nursing research. 5th ed. Philadelphia: Lippincott; 1996.

5. De Vet HCW, Terwee CB, Bouter LM. Current challenges in clinimetrics. J Clin Epidemiol 2003; 56:1137-1141.

6. Fayers PM, Machin D. Quality of life. Assessment, analysis and interpretation. Chichester (UK): John Wiley \& Sons; 2000.

7. Fitzner K. Reliability and validity. Diabetes Educ 2007; 33(5):775-780.

8. Frost MH, Reeve BB, Liepa AM, Stauffer JW, Hays $\mathrm{RD}$. What is sufficient for reliability and validity of patient-reported outcome measures? Value Health 2007; 10(Supl. 2):S94-S105.

9. Esperidião M, Trad, LAB. Avaliação de satisfação de usuários. Cien Saude Colet 2005; 10(Supl.):303-312.

10. Sireci SG. The construct of content validity. Soc Indic Res 1998; 45:83-117.

11. Roberts P, Priest H, Traynor M. Reliability and validity in research. Nurs Stand 2006; 20(44):41-45.

12. Contandriopoulos AP, Champagne F, Potvin L, Denis JL, Boyle P. Saber preparar uma pesquisa. $3^{a}$ ed. São Paulo: Hucitec, Abrasco; 1999.

13. LoBiondo-Wood G, Harber J. Nursing Research: methods, critical appraisal, $e$ and utilization. 4 th ed. St Louis: Mosby-Year Book; 1998.

14. Burns N, Grove SK. The practice of nursing research: conduct, critique \& utilization. $3^{\text {rd }}$ ed. Philadelphia: Saunders Company; 1997.

15. Mendonça KMPP, Guerra RO. Desenvolvimento e validação de um instrumento de medida da satisfação do paciente com a fisioterapia. Rev Bras Fisioter 2007; 11(5):369-376.

16. Habib ES, Magalhães LC. Criação de questionário para detecção de comportamentos atípicos em bebês. Rev Bras Fisioter 2007; 11(3):177-183.

17. Beyea S, Nicoll LH. Writing an integrative review. AORN J 1998; 67(4):877-880

18. Wynd CA, Schmidt B, Schaefer MA. Two quantitative approaches for estimating content validity. West J Nurs Res 2003; 25(5):508-518.

19. Haynes SN, Richard DCS, Kubany ES. Content validity in psychological assessment: a functional approach to concepts and methods. Psychol Assess 1995; 7(3):238-247.

20. Rubio DM, Berg-Weger M, Tebb SS, Lee S, Rauch S. Objectifying content validity: conducting a content validity study in social work research. Soc Work Res 2003; 27(2):94-105.

21. Polit DF, Hungler BP. Fundamentos de pesquisa em enfermagem. $3^{\text {a }}$ ed. Porto Alegre: Artes Médicas; 1995.
22. Polit DF, Beck CT. The content validity index: are you sure you know what's being reported? Critique and recomendationas. Res Nurs Health 2006; 29:489-497.

23. Lynn MR. Determination and quantification of content validity. Nurs Res 1986; 35(6):382-385.

24. Pasquali L. Psicometria. $2^{a}$ ed. Petrópolis (RJ): Editora Vozes; 2004.

25. DeVon HA, Block ME, Moyle-Wright P, Ernst DM, Hayden SJ, Lazzara DJ, Savoy SM, Kostas-Polston E. A psychometric toolbox for testing validity and reliability. J Nurs Scholarsh 2007; 39(2):155-64.

26. Carmines EG, Zeller RA. Reliability and validity assessment. $11^{\text {th }}$ ed. Beverly Hills (CA): Sage; 1988.

27. Grant JS, Davis LL. Selection and use of content experts for instrument development. Res Nurs Health 1997; 20(3):269-274.

28. Berk RA. Importance of expert judgment in content-related validity evidence. West J Nurs Res 1990; 12(5):659-671.

29. Kirshner B, Guyatt G. A methodological framework for assessing health indices. J Chron Dis 1985; 38(1): 27-36.

30. Pasquali L. Princípios de elaboração de escalas psicológicas. Rev Psiquiatr Clin 1998; 25(5): 206-13.

31. Streiner DL, Norman GR. Health measurement scales. A practical guide to their development and use. $2^{\text {nd }}$ ed. New York: Oxford University Press; 2002.

32. Hunt SM, Bucquet D, Niero M, Wiklund I, McKenna S. Cross-cultural adaptation of health measures. Health Policy 1991; 19(44):33-44.

33. Guillemin F, Bombardier C, Beaton, D. Cross-cultural adaptation of health-related quality of life measures: literature review and proposed guidelines. J Clin Epidemiol 1993; 46(12):1417-1432.

34. Guillemin F. Cross-cultural adaptation and validation of health status measures. Scand J Rheumatol 1995; 24(2):61-63.

35. Hutchinson A, Bentzen N, Konig-Zanhn C. Cross cultural health outcome assessment: a user's guide. The Netherlands: ERGHO; 1996.

36. Alexandre NMC, Guirardello EB. Adaptación cultural de instrumentos utilizados em salud ocupacional. Rev Panam Salud Publica 2002; 11(2):109-111.

37. Beaton DE, Bombardier C, Guillemin F, Ferraz MB. Guidelines for the process of cross-cultural adaptation of self-report measures. Spine 2000; 25(24): 3186-3191.

38. Davis LL. Instrument review: getting the most from a panel of experts. Appl Nurs Res 1992; 5(4):194-197.

39. Tilden VP, Nelson CA, May BA. Use of qualitative methods to enhance content validity. Nurs Res 1990; 39(3):172-175.

40. Hyrkäs K, Appelqvist-Schmidlechner K, Oksa L. Validating an instrument for clinical supervision using an expert panel. Int J Nurs Stud 2003; 40(6):619-625.

41. McGilton K. Development and psychometric evaluation of supportive leadership scales. Can J Nurs Res 2003; 35(4):72-86.

42. Morse JM. Approaches to qualitative-quantitative methodological triangulation. Nurs Res 1991; 40(1): 120-123. 
43. Hulley SB, Cummings SR, Browner WS, Grady D, Hearst N, Newman TB. Delineando a pesquisa clínica. 2a ed. Porto Alegre: Artmed; 2003.

44. Topf M. Three estimates of interrater reliability for nominal data. Nurs Res 1986; 35(4):253-245.

45. Wynd CA, Schaefer MA. The Osteoporosis Risk Assessment Tool: establishing content validity through a panel of experts. Appl Nurs Res 2002; 16(2):184-188.

46. Siegel S, Castellan HJ. Estatística não paramétrica para ciências do comportamento. $2^{\text {a }}$ ed. Porto Alegre: Artmed; 2006.

Artigo apresentado em 06/07/2009

Aprovado em 08/11/2009

Versão final apresentada em 29/11/2009 